\title{
ARTICLE OPEN \\ Artificial gravity field, astrophysical analogues, and topological phase transitions in strained topological semimetals
}

\author{
Shan Guan ${ }^{1,2}$, Zhi-Ming Yu ${ }^{1,2}$, Ying Liü ${ }^{2}$, Gui-Bin Liu', Liang Dong ${ }^{3}$, Yunhao Lu ${ }^{4}$, Yugui Yao ${ }^{1}$ and Shengyuan A. Yang ${ }^{2}$
}

Effective gravity and gauge fields are emergent properties intrinsic for low-energy quasiparticles in topological semimetals. Here, taking two Dirac semimetals as examples, we demonstrate that applied lattice strain can generate warped spacetime, with fascinating analogues in astrophysics. Particularly, we study the possibility of simulating black-hole/white-hole event horizons and gravitational lensing effect. Furthermore, we discover strain-induced topological phase transitions, both in the bulk materials and in their thin films. Especially in thin films, the transition between the quantum spin Hall and the trivial insulating phases can be achieved by a small strain, naturally leading to the proposition of a novel piezo-topological transistor device. Possible experimental realizations and analogue of Hawking radiation effect are discussed. Our result bridges multiple disciplines, revealing topological semimetals as a unique table-top platform for exploring interesting phenomena in astrophysics and general relativity; it also suggests realistic materials and methods to achieve controlled topological phase transitions with great potential for device applications.

npj Quantum Materials (2017)2:23; doi:10.1038/s41535-017-0026-7

\section{INTRODUCTION}

Relativity is a fundamental aspect for all elementary particles in the high-energy regime. In condensed matter physics, however, the relevant energy scale we probe is much lower (compared with, e.g., the electron rest mass), hence the electronic dynamics is usually considered as non-relativistic. Nonetheless, due to interactions with lattice and between electrons themselves, the electron properties in crystalline solids are strongly renormalized, and the resulting low-energy electron quasiparticles can behave drastically different from free electrons. Remarkably, in a class of recently discovered topological semimetal materials, the band structures feature nontrivial band-crossings close to the Fermi level, around which the low-energy quasiparticles become massless and resemble relativistic particles. For example, in socalled Weyl semimetals, the Fermi surface consists of isolated band-crossing points, each carrying a topological charge of \pm 1 corresponding to its chirality, and the low-energy quasiparticles mimic the Weyl fermions in high-energy physics. ${ }^{1,2}$ With further protection from crystalline symmetry, a pair of Weyl points can be stabilized at the same point (called the Dirac point) in the energy-momentum space, realizing the Dirac semimetal (DSM) phase. ${ }^{3} \mathrm{~A}$ number of $3 \mathrm{D}$ materials have been predicted to host Weyl/Dirac points. ${ }^{4-9}$ Some of them, including the DSMs $\mathrm{Na}_{3} \mathrm{Bi}$ and $\mathrm{Cd}_{3} \mathrm{As}_{2},{ }^{10,11}$ have been confirmed in recent experiments. ${ }^{12-19}$

These topological semimetals offer a versatile platform for simulating relativistic particles and their many fascinating phenomena. ${ }^{20-22}$ Indeed, the nontrivial topology of the bandcrossing point dictates the emergence of Lorentz symmetry for the low-energy quasiparticles: ${ }^{23-25}$ the inverse quasiparticle propagator can be written in a general and manifestly covariant form

$$
\mathcal{G}^{-1}=\sigma^{a} e_{a}^{\mu}\left(p_{\mu}-p_{\mu}^{(0)}\right),
$$

where $p_{\mu}$ is the covariant energy-momentum four-vector, $p_{\mu}^{(0)}$ corresponds to the location of the Weyl point, $\sigma^{a}=(1, \sigma)$ with $\sigma$ the vector of Pauli matrices, and all material-specific model parameters are encoded in the tetrad field $e_{\alpha}^{\mu}$. Consequently, the relativistic spectrum follows the equation:

$$
g^{\mu \nu}\left(p_{\mu}-p_{\mu}^{(0)}\right)\left(p_{\nu}-p_{\nu}^{(0)}\right)=0,
$$

where $g^{\mu \nu}=\eta^{\alpha \beta} e_{\alpha}^{\mu} e_{\beta}^{\nu}\left(\eta^{\alpha \beta}=\operatorname{diag}(-1,1,1,1)\right)$ plays the role of an effective spacetime metric and $p_{\mu}^{(0)}$ acts as an effective $U(1)$ gauge potential, constituting the "fermionic vacuum" where the lowenergy quasiparticles live. Here $g^{\mu v}$ and $p_{\mu}^{(0)}$ are fully determined by the material band structure, and their spatial and temporal variation may give rise to effective gravity and gauge fields, respectively. As a direct manifestation of the emergent relativistic symmetry, the effective spacetime metric $g^{\mu v}$ offers intriguing possibility to probe effects from general relativity in solid-state systems. Such possibility has not been explored so far.

Meanwhile, topological semimetals represent the neighbor state to various topological quantum phases. Particularly, DSMs are long believed to be an ideal platform for the systematic study of topological phase transitions (TPTs). However, a realistic approach to achieve controllable and reversible TPTs is still missing. Such an approach is much desired because of its

\footnotetext{
${ }^{1}$ Beijing Key Laboratory of Nanophotonics and Ultrafine Optoelectronic Systems, School of Physics, Beijing Institute of Technology, Beijing 100081, China; ${ }^{2}$ Research Laboratory for Quantum Materials, Singapore University of Technology and Design, Singapore 487372, Singapore; ${ }^{3}$ International Center for Quantum Materials, Peking University, Beijing 100871, China and ${ }^{4}$ School of Materials Science and Engineering, Zhejiang University, Hangzhou 310027, China

Correspondence: Yugui Yao (ygyao@bit.edu.cn) or Shengyuan A. Yang (shengyuan_yang@sutd.edu.sg)
}

Received: 16 November 2016 Revised: 12 March 2017 Accepted: 5 April 2017

Published online: 10 May 2017 
significance in both fundamental physics investigation and in potential topological device applications.

In this work, we show that both objectives mentioned above can be achieved via lattice strain in topological semimetals. As a notable advantage, the solid-state systems admit an easy tuning of their properties by strain. Taking two DSMs as concrete examples and with first-principles calculations, we demonstrate that the quasiparticle spectrum can be efficiently controlled by uniaxial strain. We show that an inhomogeneous strain profile can generate warped spacetime with fascinating analogues in astrophysics. Particularly, we analyze the possibility to simulate black-hole/white-hole event horizons and gravitational lensing effect. Furthermore, we find that a larger strain can completely change the fermionic vacuum, leading to a TPT between DSM and trivial insulator phases, during which the two Dirac points collide at the $\Gamma$ point and pair-annihilate. More importantly, for a quasi-2D DSM thin film, a small strain is sufficient to control a TPT between quantum spin Hall (QSH) and trivial insulator phases. This is regarded as a key to the realization of a topological transistor. The discovery here allows us to propose a novel piezo-topological transistor device. Thus, our work not only establishes bridges between distinct disciplines such as condensed matter physics, astrophysics, and general relativity, it also reveals realistic platform and methods to study the intriguing topological phase transitions and to achieve unprecedented device functionalities for applications.

\section{RESULTS}

Strain tuning and TPT in bulk material

In this work, we take the two DSMs $\mathrm{Na}_{3} \mathrm{Bi}$ and $\mathrm{Cd}_{3} \mathrm{As}_{2}$ as concrete examples to demonstrate our general idea. These two materials represent the first two topological semimetals that have been confirmed by experiment. ${ }^{12-15}$ We choose them as examples because they share a relatively simple low-energy bulk band structure. Both materials have a single pair of Dirac points located at $k_{z}= \pm k_{D}$ on the $k_{z}$-axis near the $\Gamma$ point of the Brillouin zone. ${ }^{10,11}$ Each Dirac point is four-fold degenerate, consisting of two Weyl points of opposite chirality. The decoupling of the two Weyl points (hence the stability of the Dirac point) is protected by the $C_{3}\left(C_{4}\right)$ rotational symmetry of $\mathrm{Na}_{3} \mathrm{Bi}\left(\mathrm{Cd}_{3} \mathrm{As}_{2}\right)$. The band ordering is inverted at the $\Gamma$ point, as it should be for the realization of bandcrossing points.

In this work, we focus on the uniaxial strain along the crystalline $c$-axis (z-direction; see Fig. 1a), which preserves the corresponding rotational symmetry hence the existence of Dirac points. The lowenergy effective model can be written for each Dirac point. For example, the quasiparticles around the Dirac point at $k_{z}=+k_{D}$ are described by two copies of the Weyl Hamiltonian: ${ }^{10,11}$

$\mathcal{H}_{ \pm}= \pm v_{\perp} k_{x} \sigma_{x}+v_{\perp} k_{y} \sigma_{y}+v_{z}\left(k_{z}-k_{D}\right) \sigma_{z}+w\left(k_{z}-k_{D}\right)$,

each with a definite chirality corresponding to the subscript of $\mathcal{H}$. Here $v_{\perp}$ and $v_{z}$ are the Fermi velocities in the $x y$-plane and along the $z$-axis, respectively (we set $\hbar=1$ ), and the last term in Eq. (3) tilts the spectrum along $k_{z}$. The model for the other Dirac point at $k_{z}=-k_{D}$ can be simply obtained from (3) by a time reversal operation.

Under moderate uniaxial strain, the form of model (3) is preserved, only the model parameters change with strain. The parameters can be evaluated by fitting the first-principles band structure. Since the two materials show qualitatively similar behavior, in the following presentation, our discussion will be mainly based on the results of $\mathrm{Na}_{3} \mathrm{Bi}$. (The first-principles calculation method and the results for $\mathrm{Cd}_{3} \mathrm{As}_{2}$ are presented in Supplementary Information.) The band structures of $\mathrm{Na}_{3} \mathrm{Bi}$ for several representative strains are shown in Fig. 1b-e. Here strain $\varepsilon=\left(\ell-\ell_{0}\right) / \ell_{0}$ where $\ell$ is the lattice parameter along $c$-axis and $\ell_{0}$ is its equilibrium value. The values of model parameters vs. strain are plotted in Fig. 2. Importantly, one observes that with compressive strain, $k_{D}$ decreases and approaches zero at a critical strain $\varepsilon_{c} \sim-6.2 \%$. This means that the locations of the two Dirac points are shifted towards the $\Gamma$ point by strain and collide with each other at $\varepsilon_{c}$. Beyond this point, the effective model (3) is no longer valid. From the first-principles band structure (Fig. 1c-e), one can see that the two Dirac points annihilate with each other and eventually a finite gap is opened in the spectrum, leading to a band insulator at large strains. In this process, the direct gap at the $\Gamma$ point, which indicates the strength of band inversion, shrinks and changes sign at $\varepsilon_{c}$, marking a reversal of band ordering around the $\Gamma$ point (from inverted ordering to normal ordering). Thus, the transition at $\varepsilon_{c}$ represents a TPT from a DSM phase to a trivial insulator phase (the change in Fermi surface topology means that it is also a Lifshitz transition). a

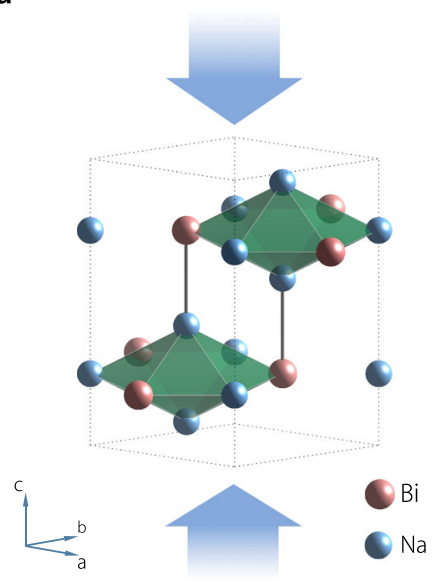

b

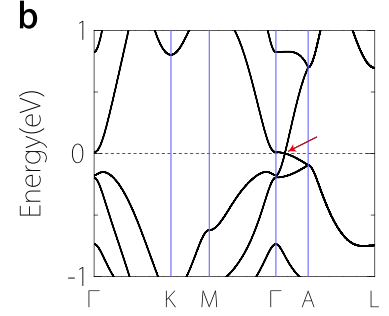

d

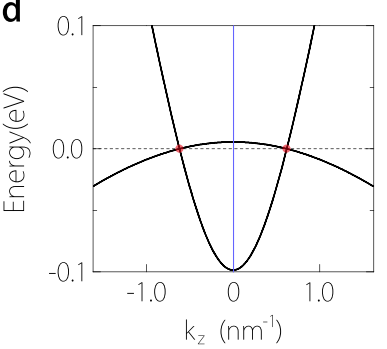

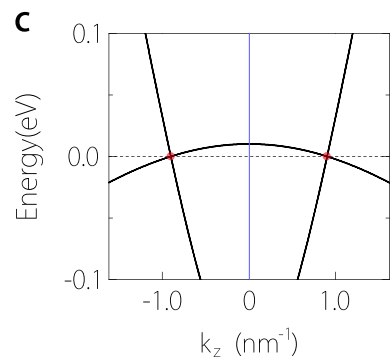

e

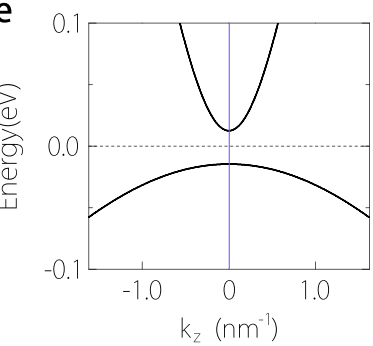

Fig. 1 First-principles band structure results for $\mathrm{Na}_{3} \mathrm{Bi}$. a Schematic representation of $\mathrm{Na}_{3} \mathrm{Bi}$ lattice structure under uniaxial strain. b Band structure without strain. c-e Enlarged band structures near the $\Gamma$ point along the $k_{z}$-axis for strain value c $0 \%, \mathbf{d}-3 \%$, and e $-7 \%$. The Dirac points are indicated by the red arrow in $\mathbf{b}$ and by red dots in $\mathbf{c}, \mathbf{d}$ 
a

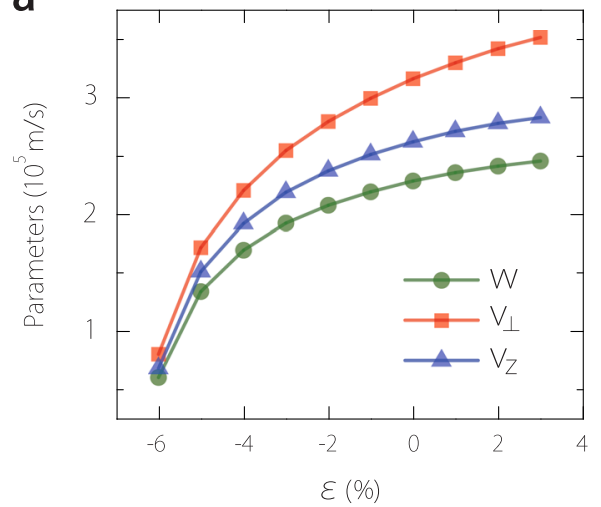

b

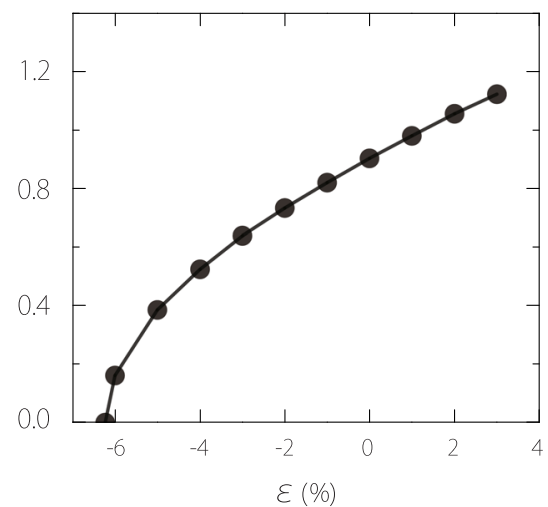

Fig. 2 Model parameter vs. strain. The model parameters $\mathbf{a} v_{\perp}, v_{z}, w$, and $\mathbf{b} k_{D}$ in Eq. (3) as a function of strain obtained by fitting the firstprinciples band structures of $\mathrm{Na}_{3} \mathrm{Bi}$

Before proceeding, we note that, first, the value of critical strain is correlated with the band inversion strength: $\mathrm{Cd}_{3} \mathrm{As}_{2}$ has a smaller inverted gap at the $\Gamma$ point than $\mathrm{Na}_{3} \mathrm{Bi}$, hence its critical strain $(\sim-1.3 \%)$ is also smaller, making the TPT comparatively easier to achieve. Second, the TPT completely changes the fermionic vacuum, from that of massless Dirac particles to massive particles with a finite excitation gap, which should be easily probed in spectroscopic or transport experiment.

Artificial gravity field and astrophysical analogues

Now let's consider the regime of small strains, for which the system lies within the DSM phase away from the TPT, such that the quasiparticles are well-described by model (3). Artificial fields are generated when we allow spacetime variation of the applied strain. Here we focus on static strain profiles and require that the strain is slowly varying on the scale of lattice constant, i.e., $|\nabla \varepsilon| \ll \ell_{0}^{-1}$, such that the strain effect can be captured by a local Hamiltonian with spatially-dependent parameters (as in Eq. (3)), forming a smooth background where the quasiparticles move around. Below, in discussing the quasiparticle propagation in the presence of a nontrivial spacetime metric, we adopt a quasiclassical description, which would further require that the strain is slowly varying compared with the Fermi wavelength $\left(|\nabla \varepsilon| \ll \lambda_{F}^{-1}\right)$.

The effective spacetime metric can be obtained by a direct comparison of model (3) with Eqs. (1) and (2). Then the coordinate differential, which characterizes the spacetime geometry, can be obtained as

$\mathrm{d} s^{2}=g_{\mu \nu} \mathrm{d} x^{\mu} \mathrm{d} x^{\nu}=-\mathrm{d} t^{2}+\frac{1}{v_{\perp}^{2}}\left(\mathrm{~d} x^{2}+\mathrm{d} y^{2}\right)+\frac{1}{v_{z}^{2}}(\mathrm{~d} z-w \mathrm{~d} t)^{2}$.

Interestingly, one observes that the tilt parameter $w$ mixes the space and time components. Its effect is like viewing an untilted spectrum (with $w=0$ ) in a moving reference frame with speed $w$. Indeed, in retrospect, one realizes that the tilt term in Eq. (3) is just the Doppler shift when (Galilean) transformed to the moving frame. With inhomogeneous static strain, the parameters $v_{\perp}, v_{z}$ and $w$ become functions of spatial coordinates. In the following, we consider two simple example configurations.

In the first example, we focus on the quasiparticle motion along the $z$-direction, assuming parameters depend only on $z$ and ignoring the $x$ and $y$ coordinates. With a general coordinate transformation $\bar{t}=t+\int{ }^{z} w\left(z^{\prime}\right) \mathrm{d} z^{\prime} /\left[v_{z}^{2}\left(z^{\prime}\right)-w^{2}\left(z^{\prime}\right)\right]$, the effective metric can be written as

$$
\mathrm{d} s^{2}=-\left[1-\left(\frac{w}{v_{z}}\right)^{2}\right] \mathrm{d} \bar{t}^{2}+\frac{1}{v_{z}^{2}} \frac{\mathrm{d} z^{2}}{\left[1-\left(\frac{w}{v_{z}}\right)^{2}\right]} .
$$

One notes that this metric shares the same form as the radial part of the familiar Schwarzschild metric for a spherical gravitating source. ${ }^{23,26}$ From the analogy, one can directly obtain the effective gravitational potential $\Phi(z)=-(1 / 2)\left(w / v_{z}\right)^{2}$ (here defined as dimensionless, as in units of $v_{z}^{2}(\infty)$ ) and the corresponding gravitational field $-(d / d z) \Phi z \hat{z}$. The analogy can be made more precise if we design the strain profile such that $\Phi(z) \propto-(1 / z)$ (which can be done in certain region excluding the $z=0$ singularity), simulating the gravity of an object located at $z=$ 0 with a mass of $-z \Phi(z) / G$, where $G$ is the Newton's constant.

The Schwarzschild metric has a coordinate singularity at the socalled Schwarzschild radius corresponding to an event horizon, where the space-like and time-like coordinates switch roles. ${ }^{26}$ One naturally speculates the possibility of similar physics here. In Eq. (5), this occurs where the value $\left|w / v_{z}\right|$ crosses 1 . The underlying physics can be easily understood as in Fig. 3a. Assuming that $v_{z}, w$ $>0$, and $w / v_{z}>1(<1)$ for $z<z_{h}\left(>z_{h}\right)$ denoted as region $A(B)$. Then from the quasiparticle spectrum, in region $B$, we have both leftand right-propagating modes; whereas in region $A$, since the tilt $w$ dominates over the Fermi velocity $v_{z}$, the spectrum is tipped over, as a result, only the right-propagating modes exist (see Fig. 3a, top panel). This means that any quasiparticle in region $A$ must cross the point $z=z_{h}$ and be emitted into region $B$. Therefore, this point represents a white-hole horizon for the quasiparticles. In addition, due to time reversal, the quasiparticles at the other Dirac point would observe $z=z_{h}$ as a black-hole horizon: a particle crosses the horizon from region $B$ to region $A$ cannot get back (see Fig. 3a, bottom panel).

For the two DSM materials considered here, we do not find the case with $\left|w / v_{z}\right|>1$ (at least for the uniaxial strain considered here). In fact, this case corresponds to so-called type-II Weyl/Dirac points, which has recently been predicted in several materials. ${ }^{27-30}$ Hence, according to our analysis, an event horizon can in principle be realized at the boundary between type-I and type-II regions. It should be noted that for type-II materials, the Fermi surface geometry completely changes, typically involving multiple electron and hole pockets. The above discussion holds only for the quasiparticles close to the Weyl/Dirac point; for quasiparticples away from the point, they will not necessarily perceive the event horizons. Nevertheless, the horizon in such case still has physical meaning as the phase boundary separating two fermionic vacua with different topology of the spectrum: with Fermi point on one side and with Fermi surface on the other side. 
a

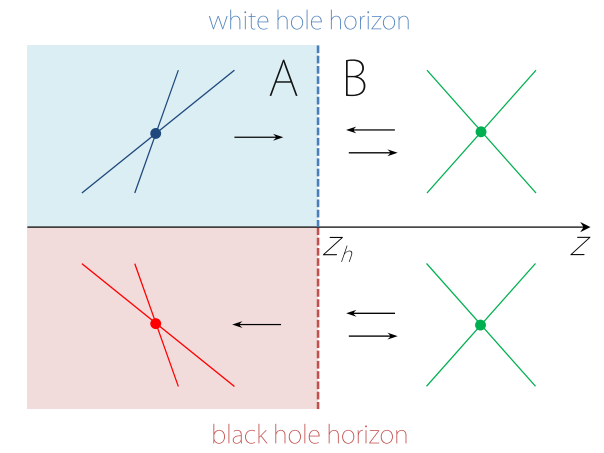

C

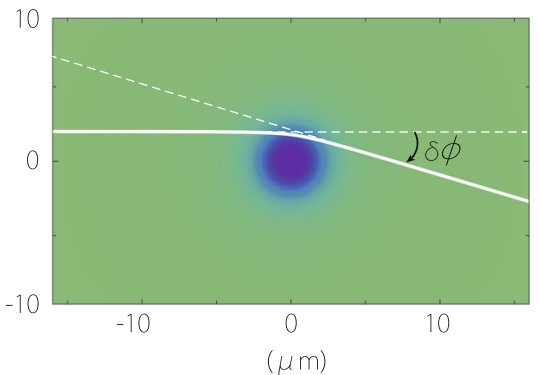

b

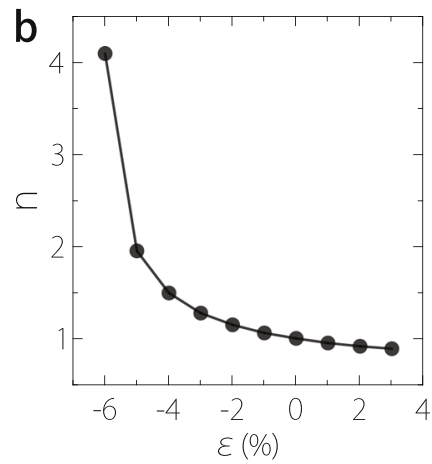

d

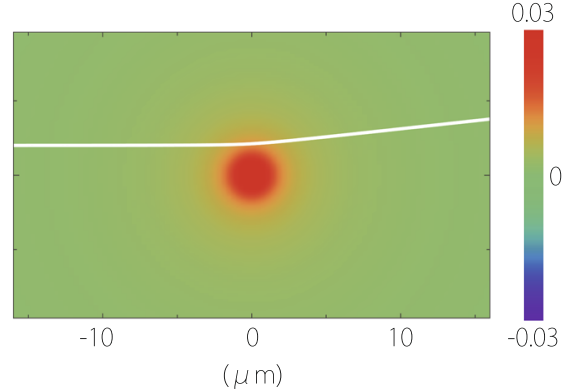

Fig. 3 Astrophysical analogues in strained topological semimetal. a Schematic figure showing (up) a white-hole horizon and (bottom) a blackhole horizon at $z=z_{h}$, corresponding to the Schwarzschild radius of metric (5). The arrows indicate the quasiparticle propagation directions in each region. b Effective refractive index $n$ for quasiparticle propagation vs. strain. c,d Analogue of gravitational lensing effect. The white line indicates a quasiparticle trajectory (geodesic) in the $x y$-plane. $\delta \phi$ indicates the deflection angle. The colormap shows the strain profile. In $\mathbf{b}-\mathbf{d}$, parameters of $\mathrm{Na}_{3} \mathrm{Bi}$ are used

In the second example, we focus on the quasiparticle motion in the $x y$-plane (ignoring the $z$-dimension) and consider the analogue of gravitational lensing effect. Then in terms of polar coordinates, we find that

$\mathrm{d} s^{2}=-\left[1-\left(\frac{w}{v_{z}}\right)^{2}\right] \mathrm{d} t^{2}+\frac{1}{v_{\perp}^{2}}\left(\mathrm{~d} \rho^{2}+\rho^{2} \mathrm{~d} \phi^{2}\right)$.

Following the standard procedure, ${ }^{26}$ one finds the quasiparticle effective "speed of light" viewed by a remote observer at $\rho=\infty$,

$c(\rho)=v_{\perp} \sqrt{1-\left(\frac{w}{v_{z}}\right)^{2}}$.

Then the propagation of the quasiparticle can be conveniently described by an effective vacuum index of refraction $n(\rho)=$ $c(\rho=\infty) / c(\rho)$, naturally leading to the bending of particle trajectories (corresponding to the geodesic in the warped spacetime) with inhomogeneous strains, like in geometric optics. One notes that here the same $n$ holds for both Dirac points. The variation of $n$ vs. strain is plotted in Fig. 3b.

To simulate the real gravitational lensing in astronomy, one can design a strain profile (using Fig. $3 \mathrm{~b}$ ), such that $n(\rho)=[1+2 \Phi(\rho)]^{-1}$ with $\Phi(\rho) \propto-\frac{1}{\rho}$ in a region excluding the singularity at $\rho=0$. Assuming the quasiparticle trajectory lies in this region and the variation of $n$ is small and smooth, the classical gravitational lensing result directly applies: ${ }^{26}$ the deflection angle $\delta \phi$ of a quasiparticle trajectory (see Fig. 3c) with the closest approaching distance $\rho_{\min }(\approx$ the impact parameter) is given by

$\delta \phi \approx-4 \Phi\left(\rho_{\min }\right)$.

Interestingly, since one can control both the vacuum state at $\rho$ $=\infty$ and the sign of applied strain, it is possible to realize $n(\rho)<1$, which corresponds to an anti-gravitating source, for which the quasiparticle trajectories are repelled from the source (Fig. 3d).
TPT in thin film and piezo-topological transistor

The TPT in the bulk typically requires a large strain (which is still within the linear elastic regime for the two materials, as shown in Supplementary Information). In the following, we show that a related TPT is more readily achievable in a DSM thin film by small strains.

First of all, one notes that in the bulk band structure of both DSMs, the gap is inverted in-between the two Dirac points, i.e., considering a $2 \mathrm{D}$ slice of the bulk Brillouin zone perpendicular to the $k_{z}$-axis, its gap is inverted if the slice lies in-between the two Dirac points, and is non-inverted otherwise. For a $\mathrm{Na}_{3} \mathrm{Bi}\left(\operatorname{or} \mathrm{Cd}_{3} \mathrm{As}_{2}\right.$ ) thin film confined in $z$-direction, ${ }^{31,32}$ the electron motion along $z$ is quantized into discrete quantum well levels, forming quantum well subbands in the spectrum. ${ }^{33,} 34$ The resulting system generally becomes semiconducting. In the quantum well approximation, ${ }^{35}$ each subband corresponds to a 2D slice in the original $3 \mathrm{D}$ band structure with an effective wave-vector $\left(\tilde{k}_{z}\right)_{m}=m \pi / L$, where the integer $m(=1,2 \ldots)$ labels the subbands and $L$ is the film thickness. Thus, the gap of the $m$ th-subband is inverted (noninverted) if $\left(\tilde{k}_{z}\right)_{m}<k_{D}\left(>k_{D}\right)$. Each inverted subband contributes a nontrivial $2 \mathrm{D} \mathbb{Z}_{2}{ }_{2}$-invariant, ${ }^{36}$ so that the quasi-2D thin film becomes a QSH insulator if there is an odd number of inverted subbands, and is a trivial insulator if this number is even. This mechanism has been revealed in the topological phase oscillation vs. $L^{11}{ }^{11,33}$

Now, under uniaxial strain, both $k_{D}$ and $\left(\tilde{k}_{z}\right)_{m}$ will change. Consider the case when the $m$ th-subband has the smallest gap and has $\left(\tilde{k}_{z}\right)_{m}<k_{D}$. With compressive strain, $\left(\tilde{k}_{z}\right)_{m}$ increases, whereas $k_{D}$ decreases. So there must exist a critical strain $\tilde{\varepsilon}_{c}$ where $\left(\tilde{k}_{z}\right)_{m}$ and $k_{\mathrm{D}}$ cross each other (see Fig. $4 \mathrm{a}$ ). In the process, the subband gap closes and re-opens with a switch of band ordering. This changes the $\mathbb{Z}_{2}$ character of the whole system by 1 , leading to a TPT between a QSH insulator and a trivial insulator phases, as illustrated in Fig. $4 \mathrm{~b}$. Since this TPT does not need $k_{\mathrm{D}}$ to vanish as 

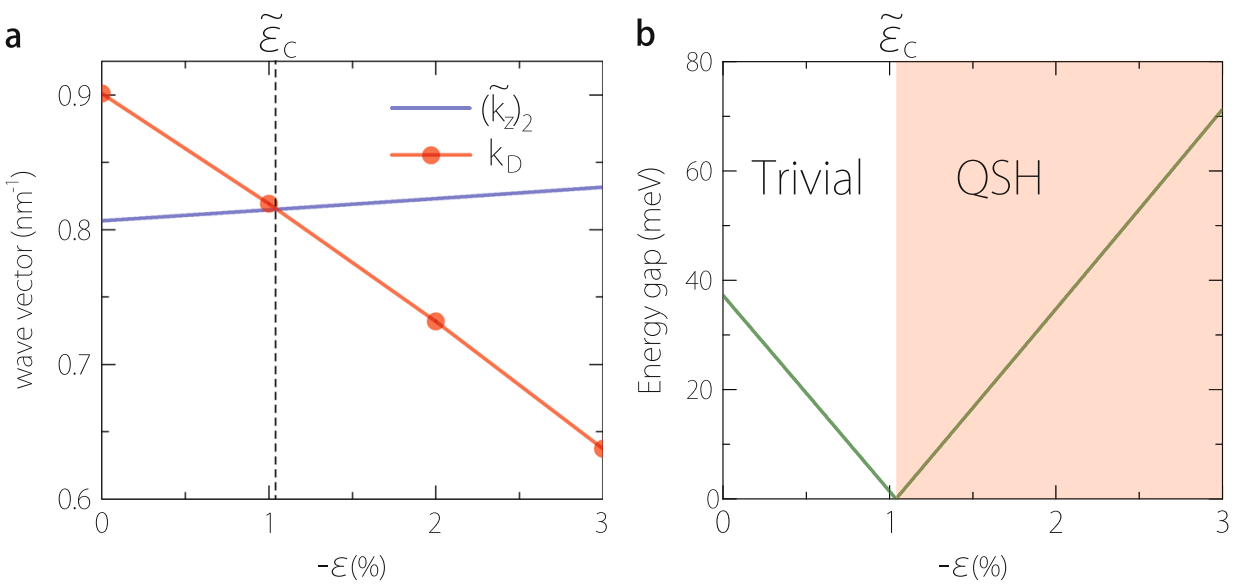

Fig. 4 Topological phase transition in thin film. a The quantum well effective wave-vector $\left(\tilde{k}_{z}\right)_{2}$ (for the second quantum well subband) and $k_{D}$ vs. strain. Their crossing-point at critical strain $\tilde{\varepsilon}_{c}$ marks a topological phase transition between a trivial insulating and a QSH insulating phases. The corresponding subband gap closes at $\tilde{\varepsilon}_{c}$, as shown in b. Here we take a $\mathrm{Na}_{3} \mathrm{Bi}$ thin film with 16-layer thickness $(\approx 78 \AA)$

a

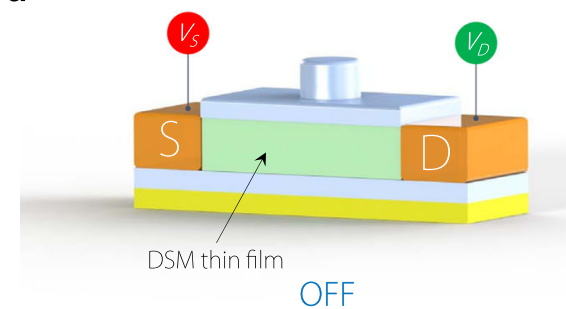

b

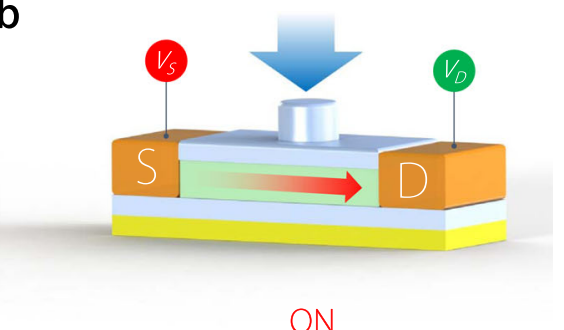

Fig. 5 Piezo-topological transistor.Schematic of a piezo-topological transistor using DSM thin film as the channel material. a The thin film is initially in the trivial insulating phase, so the transistor is in off state. $\mathbf{b}$ By applying strain to the channel region, the thin film is driven to the QSH phase with topological edge channels for conducting current. Hence the transistor is turned on. The topological channels are robust and have low heat dissipation

in the bulk case, the required strain can be much smaller (in the example shown in Fig. 4, the critical strain is $\sim 1 \%$ ).

The QSH state possesses topologically protected gapless edge channels, in which the carriers can transport without backscattering. $^{37,} 38$ This leads to the proposition of so-called topological transistor based on QSH channel materials, which is expected to have the advantages of fast operating speed, low heat dissipation, and low power consumption. ${ }^{39}$ So far, QSH state has been confirmed only in a few quantum well structures, ${ }^{37}, 38$ and how to reliably control the TPT (hence the switch between on and off states) remains a challenge for designing topological transistor. ${ }^{34}$ Our discovery here points to a promising novel device - a piezo-topological transistor. As illustrated in Fig. 5, this device has a DSM thin film as the channel. Suppose the thin film without strain is in the trivial insulator phase, for which the transistor is at the off state (Fig. 5a). By applying a small strain, the layer can be driven to the QSH phase, with current conduction through topological edge channels, corresponding to the on state (Fig. 5b). Like previous proposals, this device enjoys advantages such as low dissipation and robust operation; besides, the sensitivity to strain makes it promising for electromechanical sensing applications.

\section{DISCUSSION}

The emergence of relativistic spectrum and Lorentz invariance at low energy is a general feature dictated by the Fermi point topology. Here we take the two specific materials as examples, but the underlying physics is general and applies to other topological semimetals as well. Similar relativistic spectrum was previously discussed in the superfluid ${ }^{3} \mathrm{He}-\mathrm{A}$ phase, where analogues of black holes were suggested by controlling vortices and background superfluid flow. ${ }^{23,40,41}$ In a recent experiment, an artificial black hole for acoustic waves was simulated in an accelerating atomic Bose-Einstein condensate at sub-Kelvin temperature. ${ }^{42}$ In comparison, the solid-state system studied here can work at room temperature and permits much easier control by static means like strain or external fields, hence the predicted effects should be more readily observable.

Many experimental techniques have been developed for engineering strain, such as nanoindentation, micro-compression testing, and by using profiled substrate or stretchable substrate for thin film structures. Specifically, regarding the two examples with inhomogeneous strain profiles discussed here, the first one may be realized by using a setup similar to the micro-pillar compression test, ${ }^{43}$ and the strain variation along vertical direction could be achieved either by engineering the geometric shape of the pillar or by applying profiled lateral constriction to the side of the pillar. For the second example, an $x y$-dependent strain profile may be achieved by a setup similar to nanoindentation, e.g., by using a hard tip with engineered shape to press onto the Dirac semimetal slab. We notice that this kind of setup has recently been utilized to study the pressure-induced superconductivity in $\mathrm{Cd}_{3} \mathrm{As}_{2}{ }^{44}$ Furthermore, we point out that the lattice strain would generally be inhomogeneous in real strained samples, and more complicated strain profiles could be naturally realized. Given that the current technology can map out strain distribution with nanometer spatial resolution and with a precision $\sim 0.1 \%,{ }^{45}$ as long as the strain is slowly varying, one can compute the effective metric and study the quasiparticle propagation under artificial gravity using the idea presented here. 


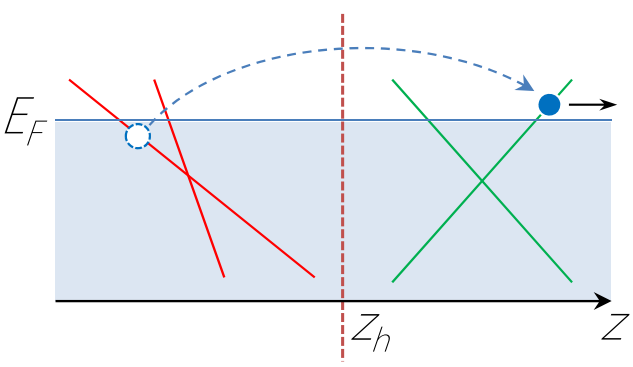

\section{black hole horizon}

Fig. 6 Analogue of Hawking radiation. Illustration of the analogue of Hawking radiation in a topological semimetal. A tunneling process due to quantum fluctuation near an artificial black-hole horizon leads to the creation of quasipartile outside the Horizon and a quasihole inside the horizon. The quasiparticle escapes from the horizon, resembling the Hawking radiation. Here $E_{\mathrm{F}}$ indicates the Fermi energy

We have mentioned that the spatial and/or temporal variation of the Weyl/Dirac point location will give rise to effective gauge fields. Here, $k_{D}$ in Eq. (3) acts as the $z$-component of a vector potential, its spatial variation generally leads to a pseudomagnetic field in the $x y$-plane. For example, for a strain varying along the $x$-direction, the induced pseudomagnetic field is along the $y$-direction, with a magnitude given by $B=\frac{1}{e} \frac{\mathrm{d} k_{D}}{\mathrm{~d} \varepsilon} \frac{\mathrm{d} \varepsilon}{\mathrm{d} x}$. For a strain variation of $3 \%$ over $10 \mathrm{~nm}$, the field strength can be up to $16.5 \mathrm{~T}$ for $\mathrm{Na}_{3} \mathrm{Bi}$. It should be noted that the field direction is reversed for the other Dirac point, as required by the time reversal symmetry. Similar pseudo-magnetic field has been studied particularly in strained graphene, ${ }^{46}$ and recently also discussed in topological semimetals. ${ }^{47,} 48$

Our treatment of the artificial gravity here is within a quasiclassical approach, which leaves out possible analogues of interesting quantum mechanical effects in curved spacetime. For example, Hawking radiation, ${ }^{49}$ the radiation of particles from the black-hole horizon stimulated by quantum vacuum fluctuation, may find analogue effects here. Assuming the presence of a blackhole horizon in a topological semimetal setting (as in Fig. 3a), the quantum tunneling process as illustrated in Fig. 6 can be interpreted as the creation of a pair of fermions due to quantum fluctuation: a quasiparticle outside the horizon and a quasihole inside the horizon. The quasiparticle escapes from the horizon, resembling the Hawking radiation. With the metric in Eq. (5) and by direct analogy, ${ }^{23}, 49$ one can find the associated Hawking temperature $T_{\mathrm{H}}=\frac{\hbar}{2 \pi k_{B}}\left|\frac{d}{d z}\left(v_{z}-w\right)\right|_{z_{b}}$ for the radiation. Nevertheless, an accurate study of these quantum effects and the methods to probe them is beyond the scope of this work and deserves a future investigation.

Finally, we note that a similar semimetal-to-insulator TPT was predicted in bulk $\mathrm{Na}_{3} \mathrm{Bi}_{1-x} \mathrm{Sb}_{x}$ and $\mathrm{Cd}_{3}\left[\mathrm{As}_{1-x} \mathrm{P}_{x}\right]_{2}$ alloys by tuning the substitute concentrations. ${ }^{50}$ In comparison, the strain approach here has the obvious advantage of allowing a reversible and continuous TPT to be achieved. For application purpose, the TPT in the thin film seems more appealing because of the dissipationless and spin-filtered transport associated with the QSH edge channels. By controlling the spatial strain profile, one can imagine to make a thin film with patterned QSH regions and trivial insulating regions, forming a topological circuit with designed 1D helical spin channels. It will provide an ideal platform for integrating various topological devices and functionalities to achieve unprecedented performance.

\section{ACKNOWLEDGEMENTS}

The authors thank D.L. Deng for helpful discussions. This work was supported by the MOST Project of China (Nos 2016 YFA0300603 and 2014CB920903), the National
Natural Science Foundation of China (Grant Nos 11574029, 11374009, 61574123, and 21373184), National Key Basic Research Program of China (2012CB825700), and Singapore MOE Academic Research Fund Tier 1 (SUTD-T1-2015004) and Tier 2 (MOE2015-T2-2-144).

\section{AUTHOR CONTRIBUTIONS}

S.G., G.-B.L., and Y.H.L. performed the first-principles calculation and the data analysis. Z.-M.Y., Y.L., L.D., and S.A.Y. performed the analytical modeling and calculation. Y.Y. and S.A.Y. supervised the work. All authors contributed to the discussion and reviewed the manuscript.

\section{COMPETING INTERESTS}

The authors declare no competing financial interests.

\section{REFERENCES}

1. Wan, X., Turner, A. M., Vishwanath, A. \& Savrasov, S. Y. Topological semimetal and fermi-arc surface states in the electronic structure of pyrochlore iridates. Phys. Rev. B 83, 205101 (2011).

2. Murakami, S. Phase transition between the quantum spin hall and insulator phases in 3D: emergence of a topological gapless phase. New. J. Phys. 9, 356 (2007).

3. Young, S. M. et al. Dirac semimetal in three dimensions. Phys. Rev. Lett. 108, 140405 (2012)

4. Burkov, A. A. \& Balents, L. Weyl semimetal in a topological insulator multilayer. Phys. Rev. Lett. 107, 127205 (2011).

5. Lu, L., Fu, L., Joannopoulos, J. D. \& Soljacic, M. Weyl points and line nodes in gyroid photonic crystals. Nat. Photon. 7, 294-299 (2013).

6. Xu, Y., Chu, R.-L. \& Zhang, C. Anisotropic Weyl fermions from the quasiparticle excitation spectrum of a 3D fulde-ferrell superfluid. Phys. Rev. Lett. 112, 136402 (2014).

7. Yang, S. A., Pan, H. \& Zhang, F. Dirac and Weyl superconductors in three dimensions. Phys. Rev. Lett. 113, 046401 (2014).

8. Weng, H., Fang, C., Fang, Z., Bernevig, B. A. \& Dai, X. Weyl semimetal phase in noncentrosymmetric transition-metal monophosphides. Phys. Rev. X 5, 011029 (2015).

9. Huang, S.-M. et al. A Weyl fermion semimetal with surface fermi arcs in the transition metal monopnictide TaAs class. Nat. Commun. 6, 7373 (2015).

10. Wang, Z. et al. Dirac semimetal and topological phase transitions in $A_{3} B i(A=N a, K$, Rb). Phys. Rev. B 85, 195320 (2012).

11. Wang, Z., Weng, H., Wu, Q., Dai, X. \& Fang, Z. Three-dimensional dirac semimetal and quantum transport in $\mathrm{Cd}_{3} \mathrm{As}_{2}$. Phys. Rev. B 88, 125427 (2013).

12. Liu, Z. K. et al. Discovery of a three-dimensional topological dirac semimetal, $\mathrm{Na}_{3} \mathrm{Bi}$. Science 343, 864-867 (2014).

13. Borisenko, S. et al. Experimental realization of a three-dimensional dirac semimetal. Phys. Rev. Lett. 113, 027603 (2014).

14. Liu, Z. K. et al. A stable three-dimensional topological dirac semimetal $\mathrm{Cd}_{3} \mathrm{As}_{2}$. Nat. Mater. 13, 677-681 (2014).

15. Jeon, S. et al. Landau quantization and quasiparticle interference in the threedimensional Dirac semimetal $\mathrm{Cd}_{3} \mathrm{As}_{2}$. Nat. Mater. 13, 851-856 (2014).

16. Xu, S.-Y. et al. Discovery of a Weyl fermion semimetal and topological fermi arcs. Science 349, 613-617 (2015).

17. Lv, B. Q. et al. Observation of Weyl nodes in TaAs. Nat. Phys. 11, 724-727 (2015).

18. Shekhar, C. et al. Extremely large magnetoresistance and ultrahigh mobility in the topological Weyl semimetal candidate NbP. Nat. Phys. 11, 645-649 (2015).

19. Yang, L. X. et al. Weyl semimetal phase in the non-centrosymmetric compound TaAs. Nat. Phys. 11, 728-732 (2015).

20. Nielsen, H. \& Ninomiya, M. The Adler-Bell-Jackiw anomaly and Weyl fermions in a crystal. Phys. Lett. B 130, 389-396 (1983).

21. Son, D. T. \& Yamamoto, N. Berry curvature, triangle anomalies, and the chiral magnetic effect in fermi liquids. Phys. Rev. Lett. 109, 181602 (2012).

22. Lundgren, R., Laurell, P. \& Fiete, G. A. Thermoelectric properties of Weyl and Dirac semimetals. Phys. Rev. B 90, 165115 (2014).

23. Volovik, G. E.The Universe in a Helium Droplet (Oxford University Press, 2003).

24. Horava, P. Stability of fermi surfaces and k theory. Phys. Rev. Lett. 95, 016405 (2005).

25. Zhao, Y. X. \& Wang, Z. D. Topological classification and stability of Fermi surfaces. Phys. Rev. Lett. 110, 240404 (2013).

26. Cheng, T.-P. Relativity, Gravitation and Cosmology (Oxford University Press, 2010).

27. Soluyanov, A. A. et al. Type-Il weyl semimetals. Nature. 527, 495-498 (2015).

28. Xu, Y., Zhang, F. \& Zhang, C. Structured Weyl points in spin-orbit coupled fermionic superfluids. Phys. Rev. Lett. 115, 265304 (2015). 
29. Ruan, J. et al. Symmetry-protected ideal Weyl semimetal in HgTe-class materials. Nat. Commun. 7, 11136 (2016).

30. Chang, T.-R. et al. Type-II topological Dirac semimetals: theory and materials prediction $\left(\mathrm{Val}_{3}\right.$ family). Preprint at http://arxiv.org/abs/1606.07555 (2016).

31. Hellerstedt, J. et al. Electronic properties of high-quality epitaxial topological Dirac semimetal thin films. Nano. Lett. 16, 3210-3214 (2016).

32. Moll, P. J. W. et al. Transport evidence for fermi-arc-mediated chirality transfer in the Dirac semimetal $\mathrm{Cd}_{3} \mathrm{As}_{2}$. Nature. 535, 266-270 (2016).

33. Xiao, X., Yang, S. A., Liu, Z., Li, H. \& Zhou, G. Anisotropic quantum confinement effect and electric control of surface states in Dirac semimetal nanostructures. Sci. Rep 5, 7898 (2015).

34. Pan, H., Wu, M., Liu, Y. \& Yang, S. A. Electric control of topological phase transitions in Dirac semimetal thin films. Sci. Rep. 5, 14639 (2015).

35. Liu, C.-X. et al. Oscillatory crossover from two-dimensional to three-dimensional topological insulators. Phys. Rev. B 81, 041307 (2010).

36. Shen, S.-Q. Topological Insulators: Dirac Equation in Condensed Matters (Springer, 2012).

37. Hasan, M. Z. \& Kane, C. L. Colloquium: topological insulators. Rev. Mod. Phys. 82 , 3045-3067 (2010).

38. Qi, X.-L. \& Zhang, S.-C. Topological insulators and superconductors. Rev. Mod. Phys. 83, 1057-1110 (2011).

39. Qian, X., Liu, J., Fu, L. \& Li, J. Quantum spin hall effect in two-dimensional transition metal dichalcogenides. Science 346, 1344-1347 (2014).

40. Unruh, W. G. Experimental black-hole evaporation? Phys. Rev. Lett. 46, 1351-1353 (1981)

41. Volovik, G. E. et al. Lifshitz transitions via the type-II Dirac and type-II Weyl points. Preprint at http://arxiv.org/abs/1604.00849 (2016).

42. Steinhauer, J. Observation of quantum Hawking radiation and its entanglement in an analogue black hole. Nat. Phys. doi:10.1038/nphys3863 (2016).
43. Jiang, L. \& Chawla, N. Mechanical properties of $\mathrm{Cu}_{6} \mathrm{Sn}_{5}$ intermetallic by micropillar compression testing. Scripta. Mater. 63, 480-483 (2010).

44. Wang, $H$. et al. Observation of superconductivity induced by a point contact on 3D Dirac semimetal $\mathrm{Cd}_{3} \mathrm{~A}_{2}$ crystals. Nat. Mater. 15, 38-42 (2016).

45. Hÿtch, M., Houdellier, F., Hüe, F. \& Snoeck, E. Nanoscale holographic interferometry for strain measurements in electronic devices. Nature. 453, 1086-1089 (2008).

46. Levy, N. et al. Strain-induced pseudo-magnetic fields greater than 300 tesla in graphene nanobubbles. Science 329, 544-547 (2010).

47. Cortijo, A., Ferreirós, Y., Landsteiner, K. \& Vozmediano, M. A. H. Elastic gauge fields in Weyl semimetals. Phys. Rev. Lett. 115, 177202 (2015).

48. Pikulin, D. I., Chen, A. \& Franz, M. Chiral anomaly from strain-induced gauge fields in Dirac and Weyl semimetals. Phys. Rev. X 6, 041021 (2016).

49. Hawking, S. W. Black hole explosions? Nature. 248, 30-31 (1974).

50. Narayan, A., Di Sante, D., Picozzi, S. \& Sanvito, S. Topological tuning in threedimensional Dirac semimetals. Phys. Rev. Lett. 113, 256403 (2014).

By Attribution 4.0 International License, which permits use, sharing, adaptation, distribution and reproduction in any medium or format, as long as you give appropriate credit to the original author(s) and the source, provide a link to the Creative Commons license, and indicate if changes were made. The images or other third party material in this article are included in the article's Creative Commons license, unless indicated otherwise in a credit line to the material. If material is not included in the article's Creative Commons license and your intended use is not permitted by statutory regulation or exceeds the permitted use, you will need to obtain permission directly from the copyright holder. To view a copy of this license, visit http://creativecommons. org/licenses/by/4.0/.

(c) The Author(s) 2017

Supplementary Information accompanies the paper on the npj Quantum Materials website (doi:10.1038/s41535-017-0026-7). 